\title{
Design and characteristics of HTSC SQUID magnetometer with a sensitivity of $10^{-13} \mathrm{THz}^{-1 / 2}$
}

\author{
V V KARTSOVNIK, P P PAVLOV, V A PAVLYUK and YU A \\ TAVRIN \\ Institute for Low Temperature Physics and Engineering, Ukr. SSR Academy of Science, \\ Kharkov 310164, USSR
}

\begin{abstract}
The results are presented of the experimental studies on forming weak links in yttrium ceramics by scribing and high-voltage discharge. The energy resolution of SQUIDs and the magnetic field sensitivity of magnetometers produced according to these methods were $6 \times 10^{-28} \mathrm{~J} / \mathrm{Hz}$ and $10^{-28} \mathrm{~J} / \mathrm{Hz}, 5 \times 10^{-13} \mathrm{~T} / \mathrm{Hz}^{1 / 2}$ and $2.5 \times 10^{-13} \mathrm{~T} / \mathrm{Hz}^{1 / 2}$, respectively. Different designs of HTSC interferometers sensitive to the external magnetic field variation are described. The factors affecting the sensitivity of r.f. HTSC SQUID-magnetometers are considered.
\end{abstract}

Keywords. HTSC SQUID; HTSC SQUID magnetometer.

\section{Introduction}

The discovery of superconductivity at a critical temperature above $90 \mathrm{~K}$ has stimulated the efforts of different research groups aimed at development of r.f. and d.c. SQUIDs (Koch et al 1987; Verkin et al 1988). The first advances gained in the implementation of both d.c. and r.f. superconducting quantum interferometers of HTSC materials made the practical application of such devices a high-priority task. Indeed, the intrinsic energy resolution of d.c. HTSC SQUIDs reaches about $10^{-29} \mathrm{~J} / \mathrm{Hz}$ (Bondarenko et al 1989), and that of r.f. HTSC SQUIDs about $10^{-28} \mathrm{~J} / \mathrm{Hz}$ (Kartsovnik et al 1988) at $77 \mathrm{~K}$. However, the absence of superconductivity magnetic flux transformers of HTSC materials does not make it possible to use the traditional solutions developed for helium-cooled SQUIDs. For the same reason, the sensitivity of r.f. SQUIDmagnetometers is more than an order of magnitude higher as compared with d.c. SQUIDs and approaches $5 \times 10^{-13} \mathrm{~T} / \mathrm{Hz}^{1 / 2}$ (Kartsovnik et al 1988). This can be explained by the fact that in the existing SQUID-magnetometers the external signals are received directly by the interferometer quantizing loop whose area is substantially large in the r.f. HTSC interferometers than in the d.c. ones.

The case of d.c. and r.f. interferometers of fabrication of various HTSC materials is stipulated by that the high- $T_{c}$ ceramics themselves already contain some weak links; this is confirmed by different studies, see for example (Eremenko et al 1989). It proved sufficient to make a constriction of tens to a few microns in a high- $T_{c}$ ceramic sample in order to demonstrate the characteristics of a single weak link. Naturally, this is connected with the difference between the available HTSC materials and classical superconductors. Otherwise, a complicated problem arises of forming a weak link with the dimensions close to $\xi$ value of $10-20 \AA$ in HTSC materials. On the other hand, the energy resolution of HTSC SQUIDs obtained to date are an oder of magnitude poorer than those estimated on the basis of the existing theories (Likharev 1986). This can be attributed to the mechanism of weak link formation in the ceramics, for these weak 
links make a major contribution to the SQUID output noise. To improve the quality of ceramics, other methods of weak link preparation are required.

This paper presents the results of our work on making r.f. HTSC SQUIDs and SQUID-based magnetometers.

\section{Weak link formation by scribing}

Our first HTSC r.f. SQUID-interferometer was made as a well-known two-hole Zimmerman device (figure 1a). For this purpose, two holes, approximately $1 \mathrm{~mm}$ in diameter, were drilled in a ceramic $\mathrm{YBa}_{2} \mathrm{Cu}_{3} \mathrm{O}_{7-x}$ block measuring $3 \times 3 \times 4 \mathrm{~mm}$ in such a way that the wall between them was $0.5 \mathrm{~mm}$ thick. A small link between the holes was then made by scribing. A resonant circuit coil was inserted into one of the interferometer holes. The resonant circuit was connected to a standard r.f. SQUID apparatus with the exciting frequency $10 \mathrm{MHz}$ (Pavlov et al 1989). A holder carrying the interferometer and the temperature sensor was cooled by liquid nitrogen or helium vapour down to $77 \mathrm{~K}$. Presently, we could observe the amplitude-frequency (AFC), current-voltage (IVC) or signal characteristics of the SQUID. As a rule, the first response observed was that of "spurious" quantizing loop, i.e. of the loop obviously formed by the link area rather than by the interferometer geometry. By successively filling up the link and observing the SQUID characteristics one can most frequently eliminate the "spurious" quantizating loop and to leave only that one which is formed by the area of interferometer proper. In this case the final link dimensions were approximately $40 \times 40 \times 40 \mu \mathrm{m}$.

The r.f. HTSC SQUID made according to the above technique had the following characteristics at $77 \mathrm{~K}$ : the resonant circuit quality factor $Q=50$, the coefficient of

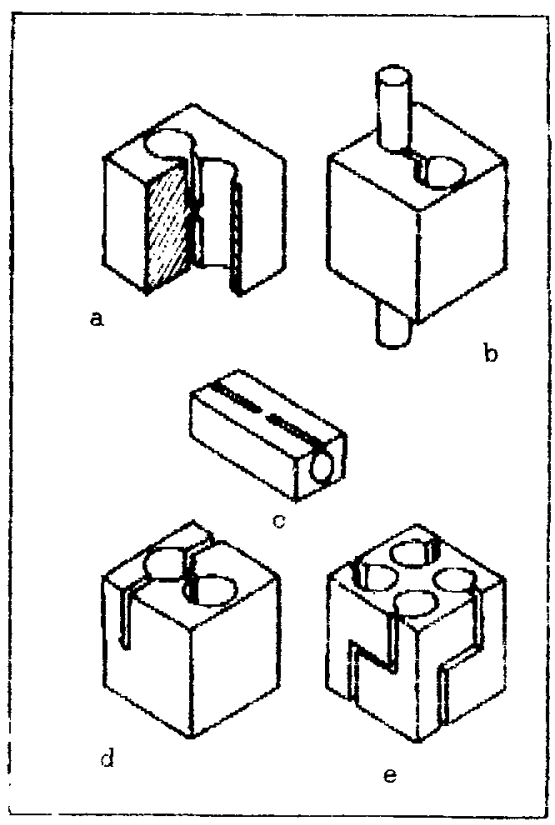

Figure 1. R.f. HTSC interferometer designs. 
resonant circuit coupling with the quantizating loop $k=0 \cdot 4$, the magnetic flux resolution $\delta \phi_{x}=2 \times 10^{-4} \phi_{0} / \mathrm{Hz}^{1 / 2}$ in the white noise region, the interferometer inductance $L_{0}=1.3 \times 10^{-10} \mathrm{H}$. The SQUID operated in the non-hysteretic mode with $l \lesssim 1$. At this time the energy resolution was $\delta \varepsilon=\left(\delta \phi_{x}\right)^{2} / 2 \cdot L_{0}=6 \cdot 10^{-28} \mathrm{~J} / \mathrm{Hz}$.

\section{HTSC interferometer designs}

As is seen, such a HTSC SQUID operating at $77 \mathrm{~K}$ has a sensitivity comparable with that of traditional r.f. SQUIDs operating at $4.2 \mathrm{~K}$. It is known that a two-hole Zimmerman interferometer does not respond to the external magnetic field variations because it is a gradiometer. Moreover, it remains a gradiometer with the holes of different diameters. Therefore, to create a magnetometer, we used a one-hole interferometer design (figure 1c). However, it turned out that at the weak link formation by scribing and at the link, the dimensions obtained there at the interferometers fail rather quickly. This was connected with the quality of our ceramics. On the other hand, a Zimmerman interferometer with a scribed link is more stable; we have a specimen operating for more than one year. For this reason, we used a two-hole interferometer to produce a HTSC SQUID-magnetometer. To make this interferometer work as a magnetometer, it is necessary to unbalance the inductance of holes and the current induced in their walls by the applied magnetic field. We therefore inserted a cylinder of HTSC material into one of the interferometer holes (figure 1b) or made a cut as shown in figure $1 \mathrm{~d}$.

The HTSC SQUID-magnetometer characteristics measured by the standard method were as follows: magnetic field period $\Delta B=2 \cdot 5 \cdot 10^{-9} \mathrm{~T}$, magnetic field resolution $\delta B_{x}=5 \cdot 10^{-13} \mathrm{~T} / \mathrm{Hz}^{1 / 2}$ in the white noise region. The boundary frequency of the white noise was about 15 to $20 \mathrm{~Hz}$. The above parameters did not vary much in SQUID-magnetometers of different design, but were strongly dependent on the HTSC ceramic material.

\section{Choice of HTSC ceramics}

To form the weak link mechanically, the ceramics should have its own intrinsic weak links. Obviously, these weak links are formed in the oxygen-depleted regions, for example at the grain boundaries. Such HTSC ceramics are characterized by low critical current density $j_{c}<500 \mathrm{~A} / \mathrm{cm}^{2}$. The investigation of critical current temperature dependence suggests the presence of S-N-S type weak link (Wang et al 1989). This is also evidenced by the low values of normal resistance $R_{N}$ of the weak link (Akimovet al 1989; Aminov et al 1989).

The S-N-S contacts with low characteristic voltages $V_{c}=I_{c} R_{N}$ restricted the sensitivity of HTSC SQUIDs. To check this assertion, we increased the r.f. SQUID exciting frequency up to $100 \mathrm{MHz}$. In this case the SQUID sensitivity remained practically unchanged which suggests that the determining contribution into the SQUID output noise comes from the weak link noise. The $1 / f$ noise is also strongly dependent on the HTSC material. The higher the critical current density of the ceramics the lower the frequency of the $1 / f$ noise onset. The presence of "spurious" quantizing loops that is peculiar to weak link preparation by scribing also deteriorates the noise characteristics of the SQUIDs. 
It should be noted that while forming a weak link by scribing, the critical current of the weak link remains practically unchanged. The link filing affects only the presence or absence of "spurious" quantizing loops in the interferometer. The critical current of the link depends on the parameters of HTSC ceramics and is to a large extent random.

Thus, to improve the HTSC SQUID characteristics, one should use the ceramics with high critical densities. However, starting with $j_{c}=700 \mathrm{~A} / \mathrm{cm}^{2}$, it is no longer possible to form a weak link by scribing.

\section{Forming a weak link by high-voltage discharge}

The main disadvantages of forming weak links by scribing is the absence of monitoring the weak link parameters and the impossibility of controlling its critical current variations. After repeated experiments, we managed to mount a set-up which was free from the above disadvantages. In this, a weak link was formed by a high-voltage discharge acting on the link of a completed assembled and rod-mounted interferometer immersed in liquid nitrogen. Initial checking of the link state and parameters of formed weak link is carried out by the AFC form of the SQUID resonant circuit. At this time, the resonant frequency variation of the SQUID circuit with the change of link resistance serves as a criterion. The intensity of high-voltage discharge effect is adjusted by changing the spark gap and the frequency of sparking.

We used as samples the one-hole interferometers with the hole diameter $1 \mathrm{~mm}$ (figure 1c) made of yttrium ceramics of different grades. The experiment shows that, at proper setting of the spark gap, this method makes it possible to vary the critical current of the weak link over a wide range from a deep hysteretic to a non-hysteretic mode of r.f. SQUID operation. In the process of weak link forming, a crater is created in the vicinity of the link; however, the link dimensions remain large enough $0 \cdot 1 \times 0 \cdot 1$ $\times 0.2 \mathrm{~mm}$ even for the ceramics with $j_{c}=700 \mathrm{~A} / \mathrm{cm}^{2}$. This can probably be explained by the fact that the superconductivity of upper layers of ceramics is disturbed.

The parameters of HTSC SQUID-magnetometer produced by this method, as measured according to the standard procedure, were as follows: quantizing loop inductance $L_{0}=2 \cdot 10^{-10} \mathrm{H}$, magnetic flux resolution $\delta \phi_{x}=10^{-4} \phi_{0} / \mathrm{Hz}^{1 / 2}$, intrinsic energy resolution $\delta \varepsilon=10^{-28} \mathrm{~J} / \mathrm{Hz}$, magnetic field period $\Delta B=2 \cdot 5 \cdot 10^{-9} \mathrm{~T}$, magnetic field resolution $\delta B_{x}=2 \cdot 5 \cdot 10^{-13} \mathrm{~T} / \mathrm{Hz}^{1 / 2}$. Figure 2 shows the IVC and signal characteristics of this SQUID.

Note the high stability of SQUID parameters during thermocycling which is obviously explained by a sufficiently massive link. As stated above, when using the same kind of ceramics for making SQUIDs with scribed weak links, the link dimensions in the operating interferometers proved to be much lesser, and they could not withstand more than two thermal cycles even at slow cooling and heating.

\section{Conclusion}

The proposed high voltage discharge technique makes it possible to form stable weak links with predetermined parameters in ceramics with any critical current density. This makes it possible to use the ceramics with $j_{c}=10^{3} \mathrm{~A} / \mathrm{cm}^{2}$ to improve the parameters of r.f. HTSC interferometers. This method considerably simplifies the interferometer manufacture and is sufficiently adaptable to streamlined production. 

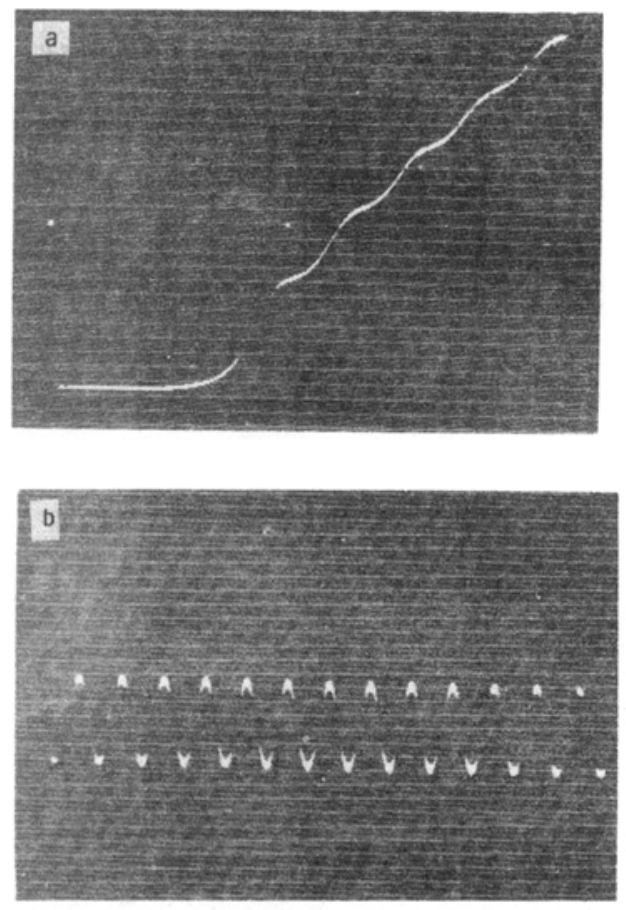

Figure 2. Characteristics of one-hole r.f. HTSC at $T=77 \mathrm{~K}$ : (a) voltage-current characteristics; (b) signal characteristics.

The sensitivity of the r.f. HTSC SQUID-magnetometers to the external magnetic field enables their application for solving a wide range of problems.

The further increase of r.f. HTSC SQUID-magnetometers sensitivity to external magnetic field may be achieved by created 4- or 8-holes interferometers (figure le).

\section{Acknowledgement}

The authors express their gratitude to N E Khebova for the provision of HTSC ceramic samples.

\section{References}

Akimov A I et al 1989 Fiz. Niz. Temp. 15535

Aminov B A et al 1989 Pisma J. Tech. Fiz. 1517

Bondarenko S I et al 1989 Sot. Superconductivity 297

Kremenko V V et al 1989 Fiz. Niz. Temp. 15695

Kartsovnik V V et al 1988 First All Union Meeting on HTSC (Kharkov) 351

Koch R H et al 1987 Appl. Physs. Lett. 51200

Likharev K K 1986 Dynamics of Josephson junction circuits (New York: Gordon and Breach)

Pavlov P P et al 1989 Geophysical Apparatus (Leningrad: Nedra) p. 3-10

Verkin B I et al 1988 Fiz. Niz. Temp. 1437

Wang S et al 1989 IEEE Trans. Magn. 25893 